\title{
Huisheng Oral Solution exerts anti-tumor effects by downregulating tissue factor and inhibiting the expression of metastasis-related factors, CD44, MMP2, and VEGF
}

\author{
Zhonghua Chen ${ }^{1,2}$, Mei Liu ${ }^{2}$, Kaiyong Xie ${ }^{3}$, Haitao Chen ${ }^{4}$ Jun Wang ${ }^{5}$, Xing Liu ${ }^{6}$ \\ ${ }^{1}$ Key Laboratory of Drug-Targeting and Drug Delivery System of the Education Ministry, Sichuan Engineering Laboratory for Plant-Sourced \\ Drug and Sichuan Research Center for Drug Precision Industrial Technology, ${ }^{2}$ Department of Pharmacology, West China School of Pharmacy, \\ Sichuan University, Chengdu 610041, China; ${ }^{3}$ Chengdu Di'ao Tianfu Pharmaceutical Group Co., Ltd. Chengdu 610041, China; ${ }^{4}$ Department of \\ Pharmacology, West China School of Pharmacy, Sichuan University; Chengdu 610041, China; ${ }^{5}$ Department of TCM Pharmacy, Chengdu Integrated \\ TCM \& Western Medicine Hospital, Chengdu 610041, China; ${ }^{6}$ Department of Orthopaedic Surgery, The Children's Hospital Chongqing Medical \\ University, Chongqing 400014, China \\ Contributions: (I) Conception and design: Z Chen, J Wang, X Liu; (II) Administrative support: K Xie, J Wang, X Liu; (III) Provision of study materials \\ or patients: All authors; (IV) Collection and assembly of data: Z Chen, M Liu, H Chen; (V) Data analysis and interpretation: Z Chen, M Liu, H \\ Chen; (VI) Manuscript writing: All authors; (VII) Final approval of manuscript: All authors. \\ Correspondence to: Xing Liu. Department of Orthopaedic Surgery, The Children's Hospital Chongqing Medical University, Chongqing 400014, \\ China. Email: liuxing322126@163.com; Jun Wang. Department of TCM Pharmacy, Chengdu Integrated TCM \& Western Medicine Hospital, \\ Chengdu 610041, China. Email: junchundi@163.com.
}

Background: This study aimed to investigate the anti-tumor effects of Huisheng Oral Solution (HSOS) on the promotion of blood circulation to dispel blood stasis, the inhibition of metastasis and inflammation, the pathogenesis of tumor progression, and to provide guidelines for using HSOS in clinical settings.

Methods: Eight-month-old Lewis lung carcinoma (LLC)-bearing $\mathrm{C}_{57} \mathrm{BL} / 6$ mice were orally administered HSOS $(0.25 \mathrm{~mL} / \mathrm{d})$ for $21 \mathrm{~d}$ starting on day 2 of model generation. One hour after the final administration, their eyeballs were dissected out to collect serum to determine tissue factor (TF) and interleukin-6 (IL-6) levels via ELISA. Blood was collected intracardially with an anticoagulant to determine fibrinogen levels, using an automated blood coagulation analyzer. The mice were euthanized via cervical dislocation and their thymi, spleens, and tumors were extracted for weight measurement and organ index calculation. CD44 and MMP2 expression and VEGF protein and mRNA expression in tumor tissues were detected using immunohistochemical (IHC) and RT-qPCR assays, respectively. Lastly, the effect of HSOS on the migration ability of A549 lung carcinoma cells was investigated using in vitro scratch assay.

Results: HSOS significantly downregulated TF and Fib in tumor-bearing mice. HSOS inhibited the overexpression of CD44, MMP2, and VEGF, and the migration ability of tumor cells. Moreover, the proinflammatory cytokine (IL-6) was significantly downregulated, but the thymic and splenic indices increased.

Conclusions: HSOS might exert anti-tumor effects by improving hypercoagulability in tumor-bearing mice, inhibiting tumor cell metastasis, alleviating inflammatory responses, and enhancing immune function.

Keywords: Huisheng Oral Solution (HSOS); Lewis lung carcinoma; hypercoagulability; tumor cell metastasis; anti-inflammation

Submitted May 28, 2019. Accepted for publication Sep 23, 2019.

doi: $10.21037 /$ tcr.2019.10.25

View this article at: http://dx.doi.org/10.21037/tcr.2019.10.25 


\section{Introduction}

Currently, malignancies are the leading cause of deaths, and they are associated with a high mortality rate (1). Malignant tumors have adverse effects on human health; hence, studies on the treatment of malignant tumors have always been considered important.

Malignant tumors are often accompanied and reinforced with hypercoagulability and metastasis or death owing to venous thromboembolism (VTE) (2-5). Moreover, tumors have been reported to be closely associated with the immune system.

Huisheng Oral Solution (HSOS) is derived from Hua Zheng Hui Sheng Dan, a classic prescription remedy of the Qing Dynasty, and is clinically reported to have significantly improved the quality of life and survival of patients who have undergone adjuvant chemotherapy over the past 21 years, especially in patients with lung cancer (6).

HSOS is a compound from 34 Chinese herbal medicines made according to the standard process stipulated in China. Its formula and component content have been fixed in strict accordance with the standards of the China pharmaceutical regulatory agency. This large compound Chinese patent medicine is characteristic of Chinese medicine. HSOS echoes the principles of Chinese medicine in the treatment of tumors. The compound has many active ingredients including Eugenol, Evodia rutaecarpa, stachydrine hydrochloride, ginsenoside, paeoniflorin, emodin, chrysophanol, etc., which have been identified according to the China National Drug Administration (CNDA). The total content of emodin $(\mathrm{C} 15 \mathrm{H} 10 \mathrm{O} 5)$ and chrysophanol (C15H10O4) should not be lower than $0.28 \mathrm{mg} / 10 \mathrm{~mL}$, according to the national drug standard [WS-10038(ZD0038)-2002-2011Z].

Early preclinical studies have reported that HSOS has an approximately $40 \%$ tumor inhibition rate (7). Considering the clinical efficacy of HSOS and recent concerns regarding the association of malignant tumors with hypercoagulability and metastasis, the present study aimed to investigate the pathogenesis of tumor progression and to provide an experimental basis for guiding the rational use of HSOS in clinical settings. We administered middle-aged Lewis lung carcinoma (LLC)-bearing $\mathrm{C}_{57} \mathrm{BL} / 6$ mice with HSOS in accordance with our previous studies. Thereafter, the antitumor effects of HSOS were investigated by determining the TF and Fib levels, which reflect the coagulation state of tumor-bearing mice and tumor cell metastasis [adhesion molecules, matrix metalloproteinases (MMPs), vascular endothelial growth factor (VEGF)], and the level of an important pro-inflammatory cytokine (interleukin-6; IL-6), which reflects immune function.

\section{Methods}

\section{Laboratory animals and cell lines}

Eight-month-old specific-pathogen-free (SPF) grade female $\mathrm{C}_{57} \mathrm{BL} / 6 \mathrm{~J}$ mice weighing $25-28 \mathrm{~g}$ were provided by Laboratory Animal Center, Sichuan University, Chengdu city, Sichuan Province, China [SCXK (Sichuan) 2013-113]. Lewis lung carcinoma (LLC) and human lung carcinoma A549 cell lines were provided by the State Key Laboratory of Biotherapy, Sichuan University. Ethics was approved by the ethics committee of West China School of Pharmacy, Sichuan University (20170128).

\section{Drugs and reagents}

The following drugs and reagents were used: HSOS (Tianfu Pharmaceutical Co., Ltd. of Chengdu Di'ao Group, Chengdu 20160216), TF Activity Assay Kit (Uscn Life Science Co., Ltd., Wuhan, 20160321), Mouse IL-6 ELISA Kit (Beijing Dakewe Biotech Co., Ltd., 20160321), Trizol Plus RNA Purification Kit (Life Technologies Corporation, USA, 15596-026), RevertAid ${ }^{\mathrm{TM}}$ First Strand cDNA Synthesis Kit (Fermentas I Thermo Fisher Scientific Inc., USA, k1622), SsoFast ${ }^{\mathrm{TM}}$ EvaGreen Supermix (BIO-RAD Laboratories, Inc., USA, 1725201).

\section{Main instruments}

The following instruments were principally used: CKX41 Inverted Microscope and 81m-25 Optical Microscope (Olympus Corporation, Japan), KEN-006 High-speed Refrigerated Centrifuge (Kendro Instrument Inc., USA), ACL-7000 Automated Blood Coagulation Analyzer (Beckman Coulter, Inc., USA), MK3 Microplate Reader (Thermo Fisher Scientific Inc., USA); ABI 7500 Real-Time PCR System (Applied Biosystems, Inc., USA), NanoDrop 2000 Ultra-trace UV Spectrophotometer (Thermo Fisher Scientific Inc., USA).

\section{Generation of the LLC mouse model}

LLC cells were resuscitated, passaged, and adjusted to $1 \times 10^{7}$ cells $/ \mathrm{mL}$ for subcutaneous inoculation in the right axilla of 
Table 1 Immunohistochemical antibody

\begin{tabular}{llll}
\hline Antibody used & CD44 & MMP2 & VEGF \\
\hline Primary antibody & HCAM antibody (sc18849) & MMP2 antibody (sc53630) & VEGF antibody (sc7369) \\
Secondary antibody & Biotinylated goat anti-rabbit IgG & Biotinylated goat anti-rabbit IgG & Biotinylated goat anti-rabbit IgG \\
& (A0277, Beyotime) & (A0277, Beyotime) & (A0277, Beyotime) \\
\hline
\end{tabular}

Table 2 The premier sequences of genes

\begin{tabular}{|c|c|c|}
\hline Primer & Forward & Reverse \\
\hline MMP2 & 5'-TGATGGCATCGCTCAGATCC-3' & 5'-GGCCTCGTATACCGCATCAA-3' \\
\hline VEGF & 5'-TGCTGTCTTGGGTGCATTGG-3' & 5'-AGGTCTCGATTGGATGGCAG-3' \\
\hline$\beta$-actin & 5'-GAGACCTTCAACACCCCAGCC-3' & 5'- AATGTCACGCACGATTTCCC-3' \\
\hline
\end{tabular}

$\mathrm{C}_{57}$ mice $(0.2 \mathrm{~mL} / \mathrm{mouse})$, and these mice were randomly divided into the model group and treatment group $(\mathrm{n}=10$ mice each). Furthermore, 10 inoculation-free mice were assigned to the normal group. Mice in the treatment group were orally administered HSOS $(0.25 \mathrm{~mL} / \mathrm{d}$ as a single dose) on day 2 of model generation for $21 \mathrm{~d}$, while mice in normal and model groups were administered physiological saline (normal saline, NS).

\section{Determination of tumor weight and inbibition rate, and thymic and splenic indices}

These mice were anesthetized for blood collection and euthanized via cervical dislocation $60 \mathrm{~min}$ after the final HSOS administration to extract their tumors, thymi, and spleens, which were then weighed to determine tumor weight index, tumor inhibition rate, and organ index as follows: tumor weight index (or organ index) $=$ tumor weight (or organ weight)/body weight after tumor removal; tumor inhibition rate $=(1-$ mean tumor weight of treatment group/mean tumor weight of model group) $\times 100 \%$.

\section{Determination of TF, Fib, and IL-6 levels}

The eyeballs of mice were dissected out to collect serum for determining TF and IL-6 expression, using an ELISA Kit in accordance with the manufacturer's instructions. Blood was collected transcardially (3.8\% sodium citrate: blood $=1: 9)$ to determine Fib levels, using an automated blood coagulation analyzer.

\section{Evaluation of CD44, MMP2, and VEGF protein expression through immunobistochemistry (IHC with ABC method) in tumor tissues}

Tumor and paracancerous tissues were fixed with $10 \%$ paraformaldehyde to and embedded in paraffin, which was then cut into sections, which were then subjected to IHC-3,3'-diaminobenzidine (DAB) staining in accordance with the manufacturer's instructions. The results were considered positive if the cytoplasm appeared brownishyellow. The mean integral optical density (IOD) value of positive cells was then determined using Image-Pro Plus 6.0 Image Processing Software (IOD $=$ area of positive region $x$ intensity). The primary and secondary antibodies used are shown in Table 1.

\section{Evaluation of CD44, MMP2, and VEGF $m R N A$ expression through RT-qPCR analysis}

Total RNA was extracted from tumor and paracancerous tissues, using the Total RNA Extraction Kit in accordance with the manufacturer's instructions. RNA samples were prepared in sections using the Trizol kit (Life Technologies Inc.). A ratio of absorbance at 260 and $280 \mathrm{~nm}\left(\mathrm{~A}_{260} / \mathrm{A}_{280}\right)$ ranging between 1.8 and 2.0 indicated high purity of RNA. The total RNA of each sample was reverse transcribed into the first strand of cDNA; its concentration was measured using an ultra-trace UV spectrophotometer. PCR amplification of cDNA was performed using primers (Table 2) in accordance with the manufacturer's instructions 
Table 3 HSOS affects the tumor weight and antitumor rate $\left(\bar{x}_{ \pm s}, \mathrm{n}=10\right)$

\begin{tabular}{lcccc}
\hline Group & Body weight $(\mathrm{g})$ & Tumor weight $(\mathrm{g})$ & Tumor weight index & Antitumor rate \\
\hline Control & $25.290 \pm 1.257$ & - & - & - \\
Model & $26.080 \pm 3.095$ & $6.251 \pm 2.574$ & $0.2355 \pm 0.0754$ & - \\
HSOS & $25.680 \pm 1.816$ & $5.106 \pm 1.516$ & $0.1961 \pm 0.0452$ & $18.32 \%$ \\
\hline
\end{tabular}

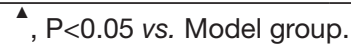

Table 4 HSOS reduces the content of TF and Fib in mices $\left(\bar{x}_{ \pm s}\right.$, $\mathrm{n}=10$ )

\begin{tabular}{lcc}
\hline Group & TF $(\mathrm{pg} / \mathrm{mL})$ & Fib $(\mathrm{mg} / \mathrm{dL})$ \\
\hline Control & $286.495 \pm 98.742$ & $98.4 \pm 32.418$ \\
Model & $470.601 \pm 155.588^{\star}$ & $249.0 \pm 41.360^{\star}$ \\
HSOS & $320.269 \pm 75.880^{\star}$ & $176.3 \pm 49.826^{\star}$ \\
\hline
\end{tabular}

${ }^{*}, \mathrm{P}<0.05$ vs. Control group; ${ }^{\wedge}, \mathrm{P}<0.05$ vs. Model group.

and expression levels of CD44, MMP2, and VEGF were normalized with that of the mouse $\beta$-actin housekeeping gene. Expression levels of each gene in paracancerous tissues were considered the controls to calculate the $\Delta \Delta \mathrm{Ct}$ value of target genes in each experimental group. The expression level of each target gene was $2^{-\Delta \Delta \mathrm{Ct}}\left[\right.$ Remarks: $2^{-\Delta \Delta \mathrm{Ct}}: \Delta \Delta \mathrm{Ct}=$ $\left.\left(\mathrm{Ct}_{\text {target }}-\mathrm{Ct}_{\text {reference }}\right)_{\text {sample to be tested }}-\left(\mathrm{Ct}_{\text {target }}-\mathrm{Ct}_{\text {reference) }}\right)_{\text {reference sample }}\right]$.

\section{In vitro scratch assay}

Human lung carcinoma A549 cells were grown until the logarithmic phase was adjusted to $3 \times 10^{5}$ cells $/ \mathrm{mL}$; the cells were then inoculated onto a 6 -well plate prior to a 24-h incubation. The cells were then treated with 250.0, $125.0,62.5$, and $31.3 \mathrm{mg} / \mathrm{mL}$ of HSOS, while cells in the blank group were treated with medium containing $0.1 \%$ DMSO. The amount of serum in the medium used in the scratch tests was $10 \%$. The in vitro scratch assay was then performed to determine the invasiveness of these cells after HSOS treatment. Three scratches were created for each well, and the plate was incubated for $24 \mathrm{~h}$. Microscopy images were randomly captured to visually assess the scratched area, using Image-ProPlus Version 6.0 software.

\section{Statistical analysis}

Numerical data were expressed as mean \pm standard deviation. One-way ANOVA was used to compare the difference among different groups, together with 1sd-ttest for post-hoc analysis. $\mathrm{P}$ values $<0.05$ were considered statistically significant. All the analysis was performed by SPSS 21.0.

\section{Results}

Effects of HSOS on the weight of transplanted LLC tumors and tumor inbibition rate

Table 3 shows that the HSOS treatment group had significantly lower tumor weight and tumor weight index than the model group, with a tumor inhibition rate of $18.32 \%$, indicating that HSOS inhibits tumor growth.

\section{Effects of HSOS on TF and Fib levels in LLC-bearing mice}

Both model and treatment groups had significantly higher TF and Fib levels than the normal group, with the treatment group showing significantly lower levels than the model group. Hypercoagulability in the tumor-bearing mice suggested that HSOS could help manage coagulopathy and fibrinolytic disorder, as shown in Table 4 .

\section{Effects of HSOS on CD44, MMP2, and VEGF protein expression in LLC-bearing mice}

IHC-DAB staining revealed that CD44, MMP2, and VEGF proteins were majorly expressed in the cytoplasm, as shown in Figure 1. Further assessment using Image-Pro Plus 6.0 Image Processing Software revealed that both model and treatment groups had significantly higher CD44-, MMP2, and VEGF-positive IOD values than the paracancerous tissue (control group), with the treatment group showing significantly lower IOD values than the model group (Table 5). Hence, HSOS treatment could reduce CD44, MMP2, and VEGF protein expression in tumor tissues. 


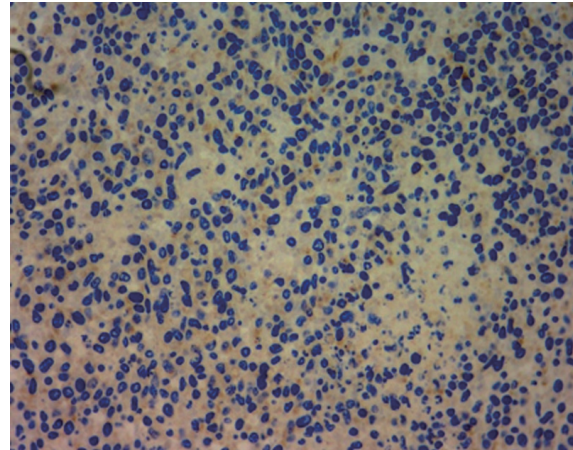

CD44 Control group

The paracancerous tissues did not express CD44.

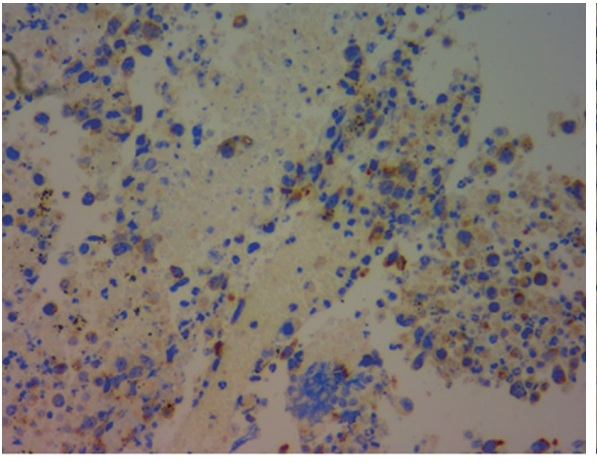

CD44 Model group

The tumor cells showing relatively high CD44 expression with a strong metastatic tumor cell adhesion.

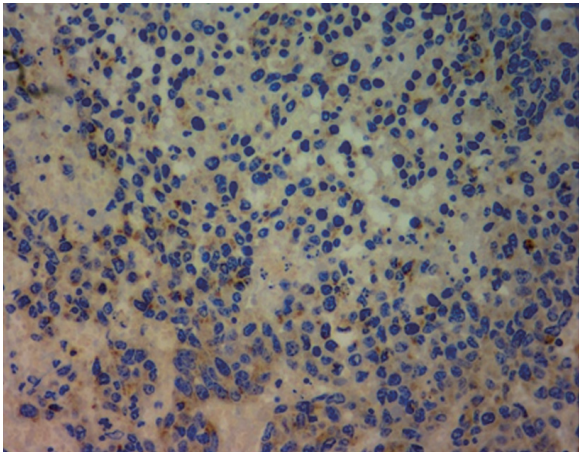

CD44 HOSO treatment group

The tumor cells showing relatively low CD44

expression than the model group, with reduced metastatic tumor cell adhesion and metastatic ability.

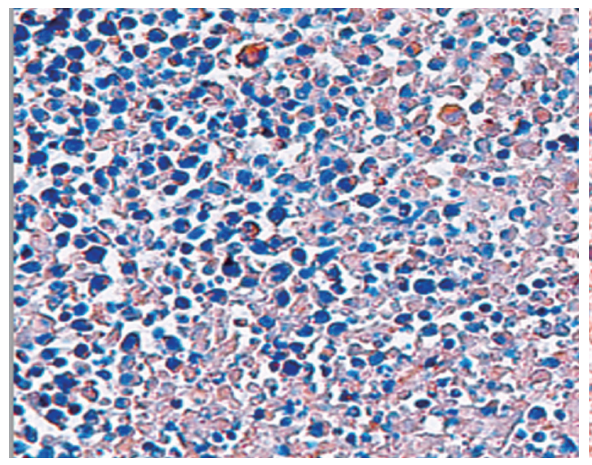

MMP2 Control group

The paracancerous tissues showing very low MMP2 expression.

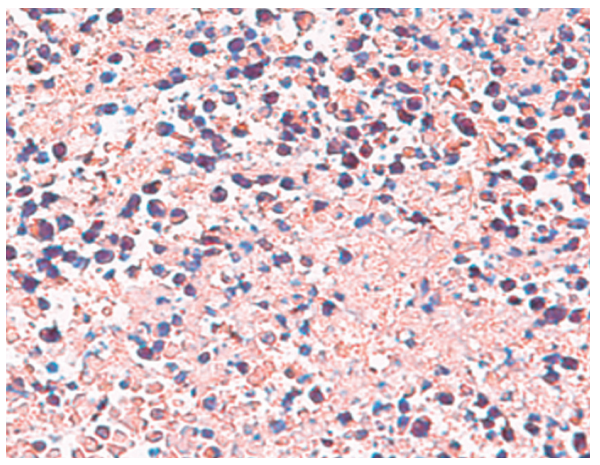

MMP2 Model group

The tumor cells showing relatively high MMP2 expression, with great migration ability and invasiveness of metastatic tumor cells.

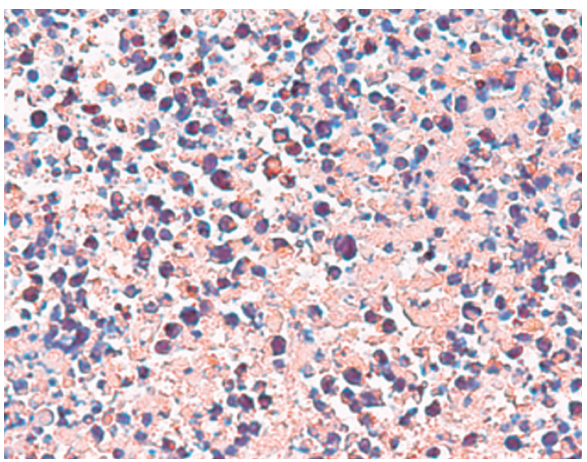

MMP2 HOSO treatment group

The tumor cells showing relatively low MMP2 expression than the model group, with reduced infiltration ability and invasiveness of metastatic tumor cells.

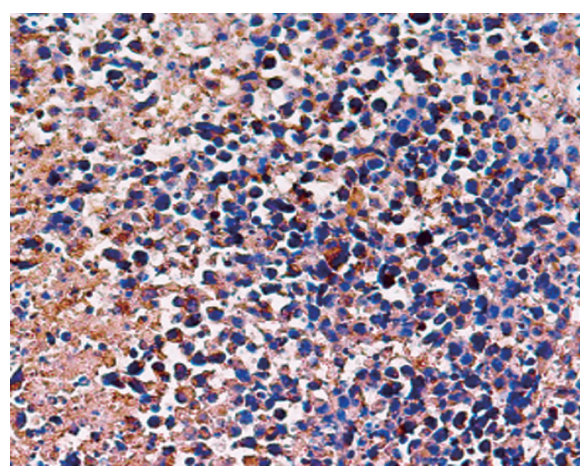

VEGF Control group

The paracancerous tissues showing relatively low VEGF expression.

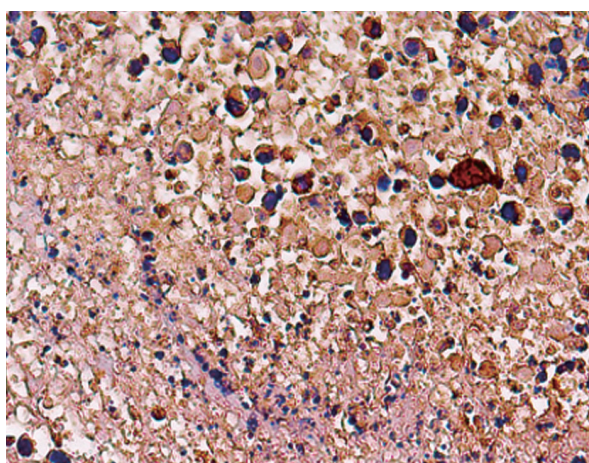

VEGF Model group

The tumor cells showing relatively high VEGF expression, with a great neovascularization and lymphatic function of tumor tissues.

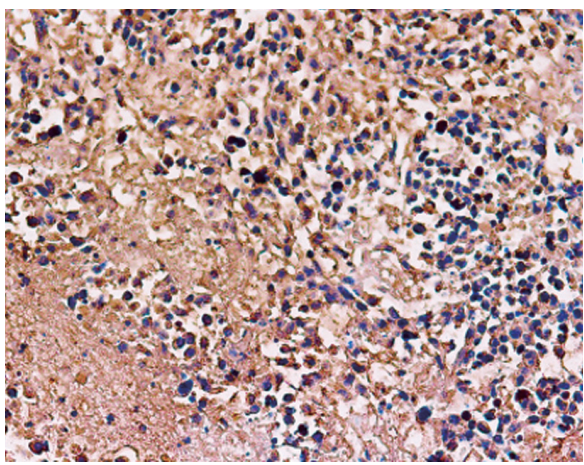

VEGF HOSO treatment group

The tumor cells showing relatively low VEGF

expression than the model group, with reduced neovascularization and lymphatic function of tumor tissues.

Figure 1 Immunohistochemical staining with antibodies to CD44, MMP2 and VEGF on tumor tissue DAB staining $\times 400$. 
Table 5 HSOS down-regulates the protein expression of CD44, MMP2 and VEGF in tumor tissue $\left(\bar{x}_{ \pm} \mathrm{s}, \mathrm{n}=10\right)$

\begin{tabular}{lccc}
\hline Group & CD44 (IOD) & MMP2 (IOD) & VEGF (IOD) \\
\hline Control & $51.28 \pm 5.17$ & $99.15 \pm 7.22$ & $87.49 \pm 7.20$ \\
Model & $121.50 \pm 7.27^{\star}$ & $147.04 \pm 6.88^{\star}$ & $168.55 \pm 7.82^{\star}$ \\
HSOS & $90.58 \pm 5.02^{\star^{\star}}$ & $122.59 \pm 5.63^{\star \mathbf{\Delta}}$ & $132.73 \pm 7.09^{\star^{\mathbf{}}}$ \\
\hline
\end{tabular}

${ }^{*}, \mathrm{P}<0.05$ vs. Control group; ${ }^{\mathbf{}}, \mathrm{P}<0.05$ vs. Model group.

Table 6 HSOS inhibits the mRNA expression of CD44, MMP2 and VEGF in tumor tissue $(\bar{x} \pm s, n=10)$

\begin{tabular}{llll}
\hline Group & CD44 & MMP2 & VEGF \\
\hline Control & $1.171 \pm 0.514$ & $1.126 \pm 0.522$ & $1.011 \pm 0.156$ \\
Model & $2.454 \pm 1.006^{\star}$ & $2.528 \pm 0.962^{\star}$ & $2.691 \pm{ }^{\star} 0.372$ \\
HSOS & $1.805 \pm 0.360^{\star \star}$ & $1.625 \pm 0.193^{\star \star}$ & $1.762 \pm 0.784^{\star \star}$
\end{tabular}

${ }^{*} \mathrm{P}<0.05$ vs. Control group, ${ }^{\star} \mathrm{P}<0.05$ vs. Model group.

\section{Effects of HSOS on CD44, MMP2, and VEGF mRNA expression in LLC-bearing mice}

Both the model and treatment groups displayed significantly higher CD44, MMP2, and VEGF mRNA expression levels than the blank control group, with the treatment group (HSOS was administered only once) showing significantly lower expression levels than the model group (Table 6).

\section{Effects of HSOS on the migration ability of tumor cells}

The treatment group displayed a significantly greater scratched area than the blank group after $24 \mathrm{~h}$ of incubation. HSOS inhibited the migration ability of tumor cells in a dose-dependent manner, as reflected by a greater scratched area (Figure 2, Table 7). In Figure 2, $0 \mathrm{~h}$ represents pictures for no medication, while $24 \mathrm{~h}$ represents pictures for $24 \mathrm{~h}$ after drug action. Table 7 describes the mean \pm standard deviation of 6 samples in different groups.

\section{Effects of HSOS on IL-6 level, thymic index, and splenic index in LLC-bearing mice}

The immune organs and IL-6 content were measured 21 days after administration. The IL-6 level was significantly higher in the treatment group than in the blank group but significantly lower than that in the model group, suggesting that HSOS treatment could reduce IL-6 levels and inhibit inflammatory responses in tumor-bearing mice (Table 8).

The tumor-bearing mice had a significantly lower thymic index and a significantly higher splenic index than the blank group. HSOS treatment improved both the thymic and splenic indices, indicating protective effects of HSOS on immune organs (Table 8).

\section{Discussion}

Our previous preliminary studies reported that mouse models generated from middle-aged-to-old mice are more susceptible to hypercoagulability and tumor cell metastasis (8). Hence, we employed 8-month-old LLCbearing $\mathrm{C}_{57} \mathrm{BL} / 6$ mice for model generation in this study. Both our previous (9.7\%) (8) and present studies $(18.3 \%)$ reported that the tumor inhibition rates of HSOS were significantly lower than $40 \%$. We had previously investigated the effects of HSOS on transplanted solid tumors of LLC and tumor-associated venous thromboembolism (VTE), using 6-8-week-old $\mathrm{C}_{57}$ mice. The experiment was performed in duplicate, and the tumor inhibition rate was approximately $40 \%$, concurrent with previous findings (7). The relatively low tumor inhibition rates found in our previous $(9.7 \%)$ and present studies (18.3\%) using middle-aged-to-old mice indicated that the reduction of tumor inhibition rate might be associated with age-associated decline in immune function in mice, which warrants further investigation.

There is a balance between coagulation and fibrinolysis in a normal body. The occurrence of a tumor will lead to imbalance, and subsequent blood hypercoagulability, which 


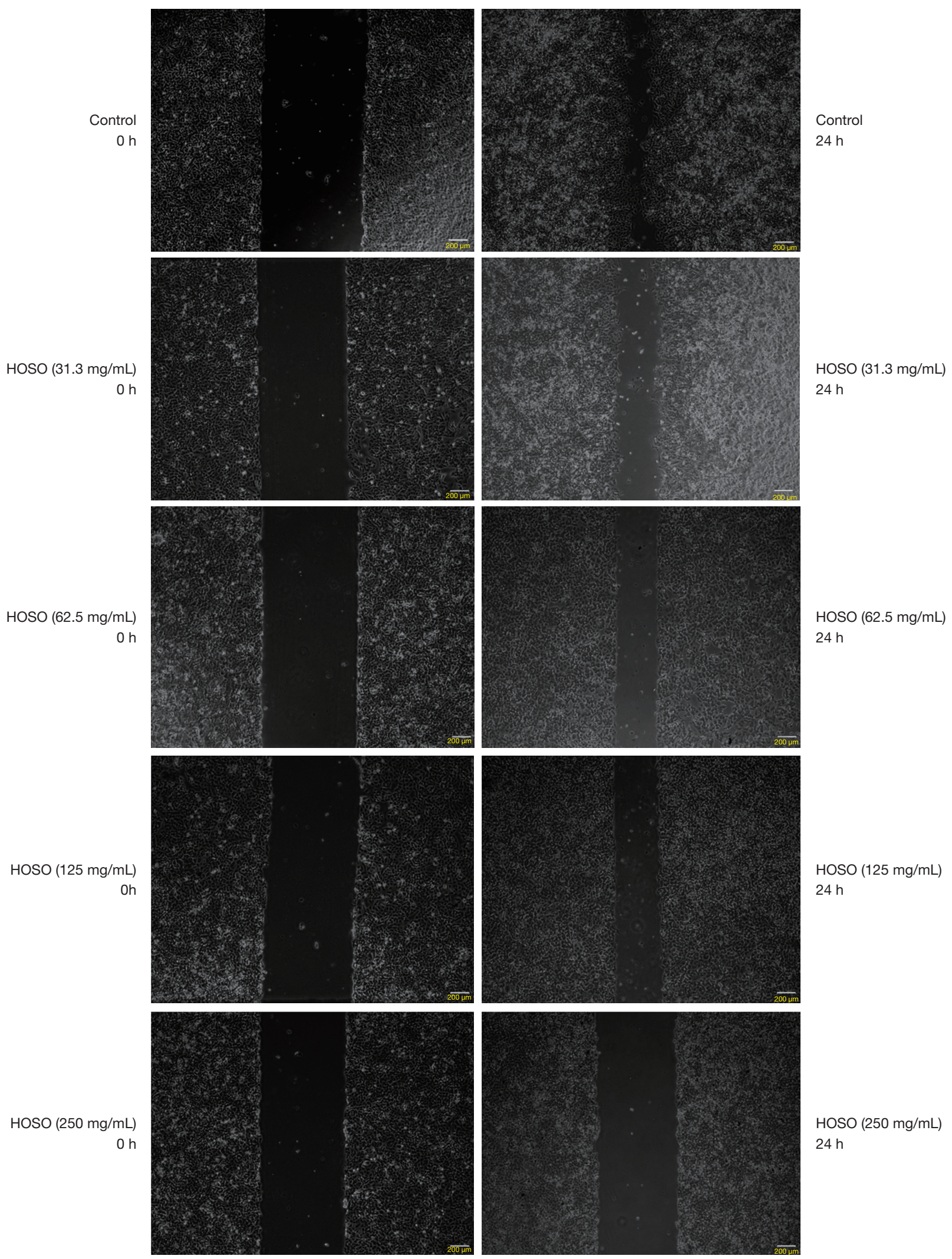

Figure 2 The impact of HSOS on the human lung cancer A549 cell's migration ( $\times 200)$. 
in turn promotes the growth and metastasis of tumor, and the formation of thrombosis. Hence, this study explored the anti-tumor mechanism of HSOS mainly through anti-hypercoagulation and anti-tumor metastasis. Antihypercoagulability indices included TF, Fib, and D-dimer levels, along with collagen-induced platelet aggregation rate. Antitumor metastasis focused on the key factors in tumor metastasis, including CD44, MMP2, and VEGF. The relationship between the observed factors in the two pathways was investigated around the HSOS. The improvement of the aforementioned factors constitutes the anti-tumor effect.

The onset of tumors disrupts the coagulationfibrinolysis balance under normal conditions, resulting in hypercoagulability, which promotes tumor growth and metastasis and thrombosis $(2,9)$. TF promotes the extrinsic coagulation pathway and its overexpression in tumor cells results in binding with Coagulation Factor VII (FVII) to form a complex that rapidly catalyzes the activation of Factor $X$, resulting in the generation of thrombin $(10,11)$. Thrombin catalyzes the conversion of Fib into insoluble fibrin, which provides structural support and facilitates the growth, infiltration, and metastasis of tumor cells (12). In this study, both TF and Fib levels reduced significantly after HSOS treatment, suggesting that HSOS could improve hypercoagulability by inhibiting TF overexpression and

Table 7 The impact of HSOS on the human lung cancer A549 cell's migration $(\bar{x} \pm s, n=6)$

\begin{tabular}{lc}
\hline Group & Scratch area (pixel) \\
\hline Control & $78,897.83 \pm 3,202.671$ \\
HSOS $(31.3 \mathrm{mg} / \mathrm{mL})$ & $153,122.17 \pm 10,838.704^{\star}$ \\
HSOS $(62.5 \mathrm{mg} / \mathrm{mL})$ & $179,653.17 \pm 5,628.069^{\star}$ \\
HSOS $(125.0 \mathrm{mg} / \mathrm{mL})$ & $20,077.500 \pm 8,329.381^{\star}$ \\
HSOS $(250.0 \mathrm{mg} / \mathrm{mL})$ & $349,301.83 \pm 9,271.637^{\star}$ \\
\hline
\end{tabular}

${ }^{*}, \mathrm{P}<0.05$ vs. Control group. reducing Fib generation.

Furthermore, tumor cells can also activate platelets, which in turn highly express $\mathrm{P}$-selectin on their plasma membranes (13). The highly expressed P-selectin mediates the adhesion of tumor cells to platelets via the binding of $\mathrm{P}$-selectin ligands on the surface of tumor cells, thereby leading to platelet aggregation and hypercoagulability (14). Subsequently, the resulting thrombosis, and cancerassociated thrombosis facilitate the evasion of immune surveillance by tumor cells (15). Additionally, the thrombi also promote distal metastasis of tumor cells by nullifying the mechanical force of normal blood flow (16). Our previous studies revealed that HSOS could reduce the rate of collagen-induced platelet aggregation (8).

Our previous studies also reported that tumor-bearing mice had significantly higher plasma D-dimer levels than the blank control group, indicating enhanced secondary fibrinolysis in tumor-bearing mice $(8,15)$. The results suggest that tumor growth not only leads to coagulationrelated abnormalities but also results in fibrinolytic disorders (17). Moreover, numerous studies have indicated that plasma D-dimer levels are associated with tumor cell metastasis (18). Briefly, the increase of plasma D-dimer levels implies an increase in blood viscosity and a reduced flow rate, which favors the adhesion of tumor cells to the vascular wall. Thereafter, the tumor cells penetrate the blood vessels, resulting in metastasis (2). Our previous studies reported that the D-dimer levels were significantly lower in the HSOS treatment group than in the model group, indicating that HSOS could indirectly inhibit tumor cell metastasis by reducing the $\mathrm{D}$-dimer levels and treating the fibrinolytic disorders in tumor-bearing mice (8).

Tumor cell metastasis during late-stage malignant tumors often results in relapse, which predominantly results in treatment failure. First, the adhesion protein CD44 promotes the adhesion of tumor cells to endothelial cells via CD44/ezrin complex formation and is thus important for the adhesion of tumor cells, which is the prerequisite

Table 8 The effect of HSOS on the content of IL- 6 and immune organs in mices $\left(\bar{x}_{ \pm \mathrm{S}}, \mathrm{n}=10\right)$

\begin{tabular}{llll}
\hline Group & \multicolumn{1}{c}{ IL-6 $(\mathrm{pg} / \mathrm{mL})$} & Thymus coefficient & Spleen coefficient \\
\hline Control & $34.418 \pm 20.253$ & $0.001960 \pm 0.000499$ & $0.002721 \pm 0.000374$ \\
Model & $66.008 \pm 19.919^{\star}$ & $0.001145 \pm 0.000267^{\star}$ & $0.012985 \pm 0.004086^{\star}$ \\
HSOS & $47.359 \pm 18.581^{\star^{\mathbf{*}}}$ & $0.001573 \pm 0.000349^{\star^{\mathbf{\Delta}}}$ & $0.016994 \pm 0.001945^{\star^{\mathbf{\Delta}}}$ \\
\hline
\end{tabular}

${ }^{\star}, \mathrm{P}<0.05$ vs. Control group; ${ }^{\mathbf{4}}, \mathrm{P}<0.05$ vs. Model group. 
of metastasis (19). Moreover, it promotes tumor cell metastasis by binding and degrading hyaluronic acid (HA) in the extracellular matrix (20). Second, the ability to degrade the extracellular matrix (ECM) and basement membrane are the prerequisites of cancer cell invasion and metastasis (21). The primary component of the ECM is Collagen IV (Col4) (22,23), which can be degraded by MMP2, similar to laminin-5, another component of the ECM. The degradation of the ECM directly disrupts the cellular barriers and triggers tumor cell metastasis, thereby promoting their migration (24). Moreover, the formation of blood and lymphatic vessels facilitates the formation of metastatic lesions from cancer cells. Many studies have shown that VEGF is upregulated in various tumors, especially in lung carcinoma, where VEGF is expressed in various stages, indicating its roles in tumor growth and metastasis (25-27). Lastly, tumor cell migration occurs throughout metastasis (28); i.e., tumor cell metastasis occurs because of their migration ability. Our study reported that HSOS downregulated CD44, MMP2, and VEGF protein, along with mRNA, in tumor tissues, thereby exerting anti-tumor effects through the multi-step, multitarget inhibition of tumor metastasis, including tumor cell adhesion, ECM degradation, tumor neovascularization, and tumor cell migration.

Inflammation is closely associated with tumors, and proinflammatory cytokines are found to be upregulated in the tumor microenvironment (TME). For instance, IL-6 has been reported to be upregulated in various tumors (29). Animal studies have reported the overexpression of IL-6 in the serum and tumor tissue of lung carcinoma-bearing mice (30). Clinical studies have also shown that lung cancer patients have significantly higher serum IL-6 levels than normal people (31). Hence, it has been proposed that IL-6 can be used as a diagnostic marker for inflammation and malignancies, with a $60-70 \%$ sensitivity and a $58-90 \%$ specificity (32). Our study also indicated that tumor growth induces overexpression of IL-6, while HSOS could regulate the inflammatory responses by downregulating IL-6, yet another possible anti-tumor mechanism of HSOS.

Current clinical studies have also reported that the onset and progression of tumors are also closely related to immune status, wherein the changes in thymic and splenic indices can be used to assess immune function (33). Many studies have reported that the onset of tumors triggers thymic atrophy and maintains the excited state of the spleen, thereby eliciting an immune response $(34,35)$. In this study, the onset of tumors reduced the mouse thymic index, which was significantly increased by HSOS. Moreover, tumors also stimulated the spleen to elicit an immune response in mice, which was further enhanced by HSOS.

This study has several limitations. We did not measure the expression levels of other interleukins/growth Factors in addition to IL6, or conduct a more complete transcriptomic profiling. We did not characterize the effect on human lung cancer cell A549 migration by interfering/neutralizing the molecular targets identified, such as VEGF, IL6, MMP2 or CD44, or by directly interfering with Huisheng activity in the in vivo experiments, in order to reach more mechanistic conclusions on the mechanism of action.

\section{Conclusions}

In summary, HSOS could inhibit tumor cell metastasis by alleviating hypercoagulability and fibrinolytic disorders through the downregulation of TF, reduction of Fib and D-dimer levels, and the inhibition of platelet aggregation. Moreover, HSOS could directly inhibit tumor metastasis by inhibiting tumor cell adhesion, ECM degradation, neovascularization, and tumor cell migration through the regulation of protein and mRNA expression of metastasisrelated factors CD44, MMP2, and VEGF. Additionally, HSOS also could inhibit inflammatory responses and enhance immune function. Together, the present results indicate that HSOS inhibits tumor growth and metastasis via multiple mechanisms.

\section{Acknowledgments}

We would like to extend our thanks to AME Editing Service for their polish of this manuscript.

Funding: None.

\section{Footnote}

Conflicts of Interest: All authors have completed the ICMJE uniform disclosure form (available at http://dx.doi. org/10.21037/tcr.2019.10.25). The authors have no conflicts of interest to declare.

Ethical Statement: The authors are accountable for all aspects of the work in ensuring that questions related to the accuracy or integrity of any part of the work are appropriately investigated and resolved. The study was conducted in compliance with the national or institutional guidelines for the care and use of animals. All procedures 
performed in studies involving human participants were in accordance with the ethical standards of the institutional and/or national research committee(s) and with the Helsinki Declaration (as revised in 2013). Individual informed consent was waived. Ethics was approved by the ethics committee of West China School of Pharmacy, Sichuan University (20170128).

Open Access Statement: This is an Open Access article distributed in accordance with the Creative Commons Attribution-NonCommercial-NoDerivs 4.0 International License (CC BY-NC-ND 4.0), which permits the noncommercial replication and distribution of the article with the strict proviso that no changes or edits are made and the original work is properly cited (including links to both the formal publication through the relevant DOI and the license). See: https://creativecommons.org/licenses/by-nc-nd/4.0/.

\section{References}

1. Lian Z, Xie Y, Lu Y, et al. Trends in the major causes of death in China, 1982-2010. Chin Med J (Engl) 2014;127:777-81.

2. Anderson JA, Witz JI. Hypercoagulable states. Crit Care Clin 2011;27:933-52.

3. Marx V. Tracking metastasis and tricking cancer. Nature 2013;494:133-6.

4. Kozlowski EO, Pavao MS, Borsig L. Ascidian dermatan sulfates attenuate metastasis, inflammation and thrombosis by inhibition of P-selectin. J Thromb Haemost 2011;9:1807-15.

5. Ekambaram P, Lambiv W, Cazzolli R, et al. The thromboxane synthase and receptor signaling pathway in cancer: an emerging paradigm in cancer progression and metastasis. Cancer Metastasis Rev 2011;30:397-408.

6. Xiong G, Zhou J, Hu YQ, et al. Huisheng Oral Solution improves quality of life of patients with advanced malignant tumors during chemotherapy. Shandong Med J 2011;51:57-8.

7. Huang GJ, Wang XM, Mao XY, et al. An experimental study of antitumor effects of Huisheng Oral Solution. Chin Trad Patent Med 1998;20:37-9.

8. Wang W, Wang H, Wang CM, et al. Treatment with Huisheng oral solution inhibits the development of pulmonary thromboembolism and metastasis in mice with Lewis lung carcinoma. Oncol Lett 2014;7:87.

9. Pavey SJ, Hawson GA, Marsh NA. Impact of the fibrinolytic enzyme system on prognosis and survival associated with non-small cell lung carcinoma. Blood Coagul Fibrinolysis 2001;12:51-8.

10. Kasthuri RS, Taubman MB, Mackman N. Role of tissue factor in cancer. J Clin Oncol 2009;27:4834-8.

11. Gerotziafas GT, Galea V, Mbemba E, et al. Tissue factor over-expression by human pancreatic cancer cells $\mathrm{BXPC} 3$ is related to higher prothrombotic potential as compared to breast cancer cells MCF7. Thromb Res 2012;129:779-86.

12. Tiedje V, Dunkler D, Ay C, et al. The role of fibrinogen plasma levels, the $-455 \mathrm{G}>\mathrm{A}$ fibrinogen and the factor XIII A subunit (FXIII-A) Val34Leu polymorphism in cancer-associated venous thrombosis. Thromb Haemost 2011;106: 908-13.

13. Furie B, Furie BC, Flaumen HR. A journey with platelet P-selectin: the molecular basis of granule secretion, signalling and cell adhesion. Thromb Haemost 2001;86:214-21.

14. Thomas GM, Panicotdubois L, Lacroix R, et al. Cancer cell-derived microparticles bearing P-selectin glycoprotein ligand 1 accelerate thrombus formation in vivo. J Exp Med 2009;206:1913-27.

15. Kröger K, Weiland D, Ose C, et al. Risk factors for venous thromboembolic events in cancer patients. Ann Oncol 2006;17:297-303.

16. Palumbo JS, Talmage KE, Massari JV, et al. Platelets and fibrin(ogen) increase metastatic potential by impeding natural killer cell-mediated elimination of tumor cells. Blood 2005;105:178-85.

17. Mackman N, Tilley RE, Key NS. Role of the extrinsic pathway of blood coagulation in hemostasis and thrombosis. Arterioscler Thromb Vasc Biol 2007;27:1687-93.

18. Suega K, Bakta IM. Correlation between clinical stage of solid tumor and D-dimer as a marker of coagulation activation. Acta Med Indones 2011;43:162-7.

19. Harrison GM, Davies G, Martin TA, et al. Distribution and expression of CD44 isoforms and Ezrin during prostate cancer-endothelium interaction. Int J Oncol 2002;21:935-40.

20. Jothy S. CD44 and its partners in metastasis. Clin Exp Metastasis 2003;20:195-201.

21. Duner S, Loparko-Lindman J, Ansari D, et al. Pancreatic cancer: the role of pancreatic stellate cells in tumor progression. Pancreatology 2010;10:673-81.

22. Matsuyama Y, Takao S, Aikou T. Comparison of matrix metalloproteinase expression between primary tumors with or without liver metastasis in pancreatic and colorectal 
carcinomas. J Surg Oncol 2002;80:105-10.

23. Kessenbrock K, Plaks V, Werb Z. Matrix

metalloproteinases: regulators of the tumor micro environment. Cell 2010;141:52-67.

24. Giannelli G, Falk-Marzillier J, Schiraldi O, et al. Induction of cell migration by matrix metalloprotease- 2 cleavage of laminin-5. Science 1997;277:225-8.

25. Lu Q, Liu J, Zhu X, et al. Expression of nerve growth factor and hypoxia inducible factor-1a and its correlation with angiogenesis in non-small cell lung cancer. J Huazhong University Sci Technol 2014;34:359-362.

26. Ming SH, Sun TY, Xiao W, et al. Matrix metalloproteinases-2,-9 and tissue inhibitor of metalloproteinase- 1 in lung cancer invasion and metastasis. Chin Med J (Engl) 2005;118:69-72.

27. Ishihara $Y$, Nishikawa T, Iijima H, et al. Expression of matrix metalloproteinase, tissue inhibitors of metalloproteinase and adhesion molecules in silicotic mice with lung tumor metastasis. Toxicol Lett 2003;142:71-5.

28. Hood JD, Cheresh DA. Role of integrins in cell invasion and migration. Nat Rev Cancer 2002;2:91-100.

29. Bromberg J, Wang TC. Inflammation and cancer: IL-6

Cite this article as: Chen Z, Liu M, Xie K, Chen H, Wang J, Liu X. Huisheng Oral Solution exerts anti-tumor effects by downregulating tissue factor and inhibiting the expression of metastasis-related factors, CD44, MMP2, and VEGF. Transl Cancer Res 2019;8(7):2602-2612. doi: 10.21037/tcr.2019.10.25 and STAT3 complete the link. Cancer Cell 2009;15:79-80.

30. Gong WY. Effect of Flavonoid Components in Scutellaria Baicalensis on Nicotine-induced Lewis Lung Cancer in Mice and Its Mechanisms. Shanghai: Doctoral Dissertation of Fudan University, 2013.

31. Zhao FQ, Liu TM, Li H, et al. Study on the content of IL-6, TNF-Q and the activity of T subsets in peripheral blood of patients with lung cancer. Chin J Lab Diogn 2001;5:83-4.

32. Hodge DR, Hurt EM, Farrar WL. The role of IL-6 and STAT3 in inflammation and cancer. Eur J Cancer 2005;41:2502-12.

33. Lopez DM, Charyulu V, Adhins B. Influence of breast cancer on thymus function in mice. J Mammary Gland Biol Neoplasia 2002;7:191-9.

34. Zhang C, Jin ZH, Song CS. Anti-tumor Effect of Water Extract from Manchurian Walnut. World Chinese Med 2010;5:210-2.

35. Yang Y, Ji HF, Wang B, et al. Effects of pine cone extract on S180 sarcomas growth, thymus and spleen indices in mouse. J Pract Oncol 2011;25:212-4. 